\title{
Mobile Ad hoc Networks: Modelling, Application and Data Routing Evaluation
}

\author{
Aadri Alaa*, Idrissi Najlae \\ University of Sultan Moulay Slimane, Faculty of Sciences and Techniques \\ Information Processing Decision Support Laboratory \\ P.B. 523, Beni Mellal, Morocco
}

\section{Article Info \\ Article history: \\ Received Jan 15, 2017 \\ Revised Feb 25, 2017 \\ Accepted Mar 17, 2017}

\section{Keywords:}

IoT

MANET

Modelization

Performance analysis

Routing protocols

\begin{abstract}
A Mobile Ad hoc Network generally called MANET[1][2] consists of a set of mobile nodes equipped with wireless communication interfaces, forming a network without resorting to any fixed infrastructure or centralized administration, this research topic has attracted increasing interest among researchers given their characteristics and potential applications and subsets (VANET[3], WSN[4]). This paper treats both scientific and technological research aimed at presenting an in-depth study of these networks and evaluating solutions to address their problems, particularly that of routing, in order to facilitate their deployment.
\end{abstract}

Copyright $@ 2017$ Institute of Advanced Engineering and Science. All rights reserved.

\section{Corresponding Author:}

Aadri Alaa,

University of Sultan Moulay Slimane, Faculty of Sciences and Techniques

Information Processing Decision Support Laboratory

P.B. 523, Beni Mellal, Morocco

Email: aadri.alaa@gmail.com

\section{INTRODUCTION}

The latest developments in the fields of information technology and telecommunications have led to a massive deployment of ad hoc wireless networks that have become a key of the Internet of Things (IoT), the nodes in these networks can be deployed to capture, store, process, and transfer environmental data permanently and also between physical contexts and virtual universes.

The IoT is often associated with machine-to-machine communication (M2M), where objects are often referred as intelligent or smart. The appearance of this concept has been an important step in facilitating implementation of solutions and applications based on sensor networks that can supervise and analyze complex systems and help in operational decision-making.

In this work, we present an overall view on the main research axes of MANETs[1][2], we discuss the subsets of these networks, their characteristics and limitations, we focus in particular on the data routing schemes that ensure the flow of packets between the different mobile units and we also expose a comparative study between the routing protocols cited in the literature.

\section{PRESENTATION OF MANETS}

\subsection{Definition and modelization of MANETs}

Ad hoc networks have the advantage of providing wireless communication between heterogeneous devices equipped with wireless transmitters and receivers using antennas that can be omnidirectional (broadcast), highly directional (point To-point), or a combination of these two types. 
In a mathematical concept an ad hoc network, in an instant $t$, can be represented by an undirected graph $G_{t=}\left(V_{t}, E_{t}\right)$, where:

$V_{t}$ : represents the set of the nodes.

$\mathrm{E}_{\mathrm{t}}$ : represents the set of links between these nodes.

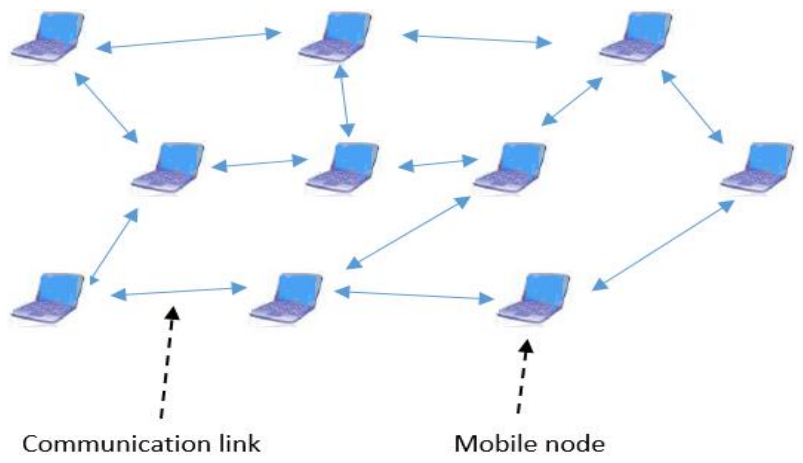

Figure 1. Mobile Ad Hoc Networks Architecture

Each mobile node (i) constitutes independently its set of multipoint relays, its one-hop neighbors are represented by:

$N^{1}=\{j \in V:(i, j) \in E\}$

While the set of neighbors at two-hop is designated by:

$$
N^{2}(i)=\coprod_{j \in N^{1}(i)} N^{1}(j)-N^{1}(i)-\{i\}
$$

The degree of the vertex $\mathrm{i}$ which designates the number of arcs that have the node $\mathrm{i}$ for origin or for destination is denoted by:

$$
d(i)=\left|\left\{x \in N^{1}(i):(i, x) \in E\right\}\right|
$$
loss of links.

This topology of MANETs can change frequently due to the mobility of the nodes which can lead to

\subsection{Characteristics of MANETs}

MANETs [1][2] have random and dynamic topologies, their objective is to form a routing infrastructure through the nodes, these networks engender new characteristics that are specific to the mobile environment:

Limited bandwidth: one of the most important characteristics of wireless-based networks is the use of shared communication media (radio waves), and that limits the bandwidth reserved for a host.

a. Dynamic topology: MANETs[1][2] topology changes frequently and rapidly because of the permanent arbitrary displacement of mobile units.

b. Limited physical security: being based on wireless communications, MANETs[1][2] are more sensitive to physical threats and attacks on the transmitted data. Moreover, the conventional techniques used to deal with these attacks are no longer applicable in ad hoc networks due to their resource limitations (memory and computing power).

c. Interference: radio links are not isolated and therefore transmissions using the same frequency or near frequencies may interfere.

d. Radio transmission errors: these errors are frequent due to the volume of data and to the limited physical characteristics of these networks.

e. Energy constraints: in some subsets of MANETs[1][2], like in the Wireless Sensor Networks (WSN)[4], mobile nodes are powered by autonomous energy sources such as batteries or other consumable sources, hence the need to set optimization criteria for the energy parameter in any control made by the system. 


\subsection{Application of MANETs}

In general, MANETs[1][2] are used in applications where the deployment of a wired network infrastructure is too constraining (cost or installation time). These applications cover a very broad spectrum, including industrial applications, data exchanges, military applications for security of services, parallel databases, distance learning, distributed file systems and distributed computing applications.

\section{THE PRINCIPAL SUBSETS OF MANETS}

\subsection{Wireless Sensor Networks (WSN)}

Wireless sensor networks[4] represent a subset of MANETs, they offer interesting application perspectives and allow to collect information from large and inaccessible environments by the use of nodes often randomly deployed with a primary constraint which is the energy limited to the power of the battery that defines their lifetime, hence the need of minimizing energy consumed in the operations of capturing, processing and sending information via radio waves to guarantee the survival of the network and its overall functioning.

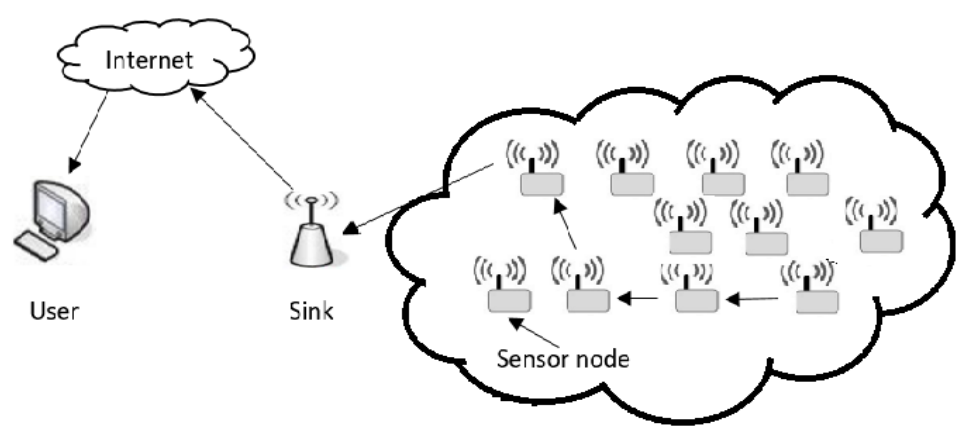

Figure 2. Modelization of Wireless Sensor Networks (WSN)

A sensor network consists essentially of a set of distributed or localized sensors, a wireless interconnection network, a central point called a sink deployed with a set of IT resources to assure data processing, query analysis and datamining. The sink communicates with the end user via Internet or satellite or any type of wireless network (such as Wi-Fi, mesh networks, WiMax, etc.). These networks can be used in several applications: fire detection in forests, airspace surveillance, medicine, connected objects, ...

According to the organization of sensors in the deployment field, the WSN can be presented under two main topologies:

a. Flat topology: in this topology, all the nodes are homogeneous and identical in terms of capacity and characteristics except the sink which is responsible of the collected data transfer to the end user. This topology allows high fault tolerance but it suffers from low scalability.

b. Hierarchical topology: in this topology, the nodes are divided into several levels of organization and responsibility. The clustering is one of the most used methods which aims to divide the network into clusters composed of a Cluster Head $(\mathrm{CH})$ and its cluster members that transfer their collected data for aggregation and transmission to the base station (BTS). This topology increases the scalability of the system, but it causes Cluster Heads overload and an unbalance in the energy consumption on the network.

\subsection{Vehicular AD hoc Networks (WSN)}

These networks are a subset of MANETs[1][2] intended to enable inter-vehicle communication and exchange of information using a specific transmission power and several channels of different rates without the need of a fixed infrastructure, the nodes in these networks are intelligent vehicles equipped with calculators, network interfaces and sensors that collect information. These networks differ from the WSNs[4] by the fact that they don't suffer from any limits in memory, processing and energy capacities.

The proposed applications for VANETs[3] require multi-hop communication where the links between vehicles are dynamic and unstable. 
VANET are intended to offer several services :dynamic flow control, alternative route guidance, access to the Internet and information services by using two types of communication:

a. Vehicle to Vehicle mode (V2V)[3][4]: a vehicle can communicate directly with another vehicle in its radio zone or through a multi-hop routing protocol using relays. This mode is characterized by a small latency and a large transmission rate.

b. Vehicle to Infrastructure mode (V2I)[3][4]: a vehicle can communicate with Road Side Units (RSUs) deployed on routes to allow access to Internet and information exchange in the network.

\section{ROUTING IN MANETS}

After describing MANETs[1][2], their main characteristics and limitations, we present the problem of routing and we describe the principles of the most cited routing protocols in the literature in order to understand the strategies and approaches used in their conception.

Data routing is not limited to finding paths between the information source nodes and the destination nodes but to finding an optimal path and ensuring a good quality of service(QoS). When the destination node is in the radio range of the source node, no routing protocol will be used, however in most applications all nodes in the network must participate in packets routing in a multi-hop environment.

Depending on the strategies and mechanisms used in data routing, routing protocols can be classified into four main classes:

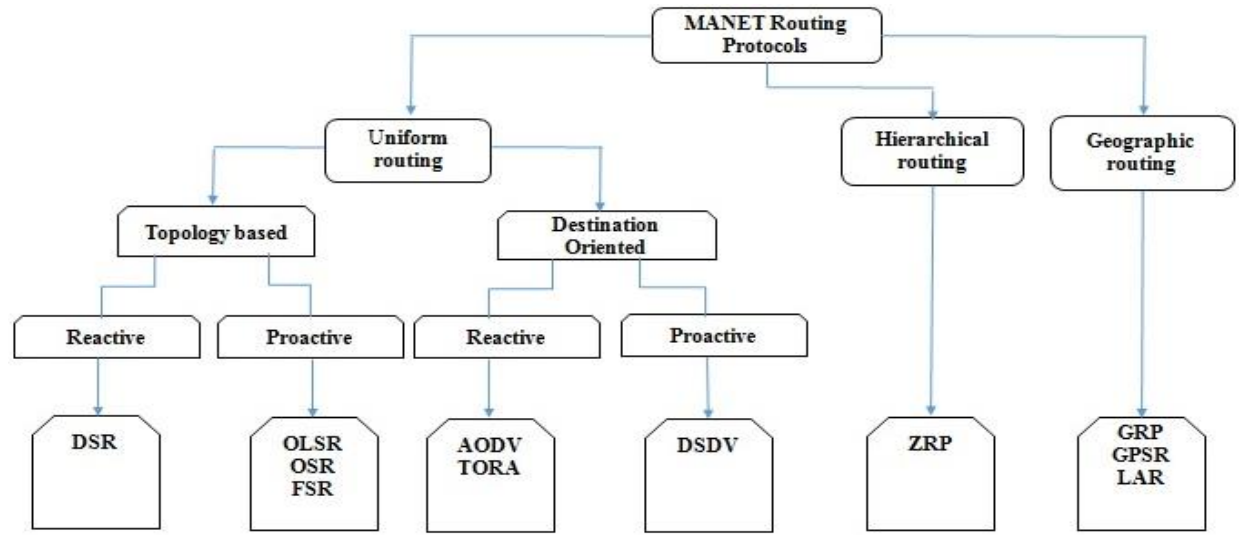

Figure 3. Classification of MANETs routing protcols

\subsection{Proactive Routing Protocols}

In this category of protocols, route management (creation and maintenance) is carried out periodically even in the absence of traffic in the network according to two main methods:

a. The Link state method where each node keeps a complete view of the entire network through periodic queries examining the state of connections with neighbors.

b. The Distance vector method where each node diffuses its neighbors vision of the distances that separate it from all the other nodes in the network and which will be useful in finding the shortest path to any destination.

Examples of proactive protocols: OLSR (Optimized Link State Routing) 11], DSDV (DestinationSequenced Distance-Vector)[11].

\subsection{Reactive Routing Protocols}

In this category, road control is done on demand (when a source wants to send a packet to a destination). Two methods are the most used:

a. The backward learning: the source node performs a flooding operation of its message throughout the network and each intermediate node (belonging to the path) indicates the path to this node by saving the route in the transmitted table, as soon as the packet is received, the destination node transmits its response as a request by creating a full duplex path.

b. Routing to the source: the source node specifies the nodes through which the message must pass to arrive at its destination by including in the header a source route, before retransmitting this 
packet each node through which the message transits deletes this header until the packet arrives to the destination where it will be delivered to the network layer of the last host.

Examples of reactive protocols: AODV (Ad hoc On-demand Distance Vector)[6][11], DSR (Dynamic Source Routing)[13][14].

\subsection{Hierarchical Routing Protocols}

To These previous categories of MANETs routing protocols, some protocols have an additional feature, they create or impose a hierarchy on the network which consists on overlaying to the physical topology a logical topology for routing where nodes are organized into groups called clusters.

Example of hierarchical protocols: ZRP (Zone Routing Protocol)[13]

\section{4 . Geographic Routing Protocols}

Geographic routing is based on the position and the use of geographic coordinates (provided by GPS) to find a path to the destination. These nodes coordinates are included in the packet to send, and then used by the intermediate nodes along with those available in their routing tables to retransmit the packet until it reaches the destination. Network management becomes simple with the node location based topology, the disadvantage is that each node must know the location of other neighboring nodes.

Example of geographic protocols: GRP (Geographic Routing Protocol)[16]

\section{SIMULATION AND PERFORMANCE METRICS}

\subsection{Research Environment}

The simulation represents the perfect way to evaluate the performance of Ad hoc routing protocols, in this research we used OPNET Modeler 14.5 (Optimum Performance NETwork)[9] which is a very powerful simulator for the network performance evaluation, it has a graphical environment and a rich library in all protocols layers. In addition to these benefits we have chosen to use this simulator to develop our node model, for the following reasons:

a. Modeling the network during the design phase and visualizing problems that occur.

b. Reproducing the structure of the network using the GUI and the object-oriented modeling.

c. Opnet simulator uses a radio propagation model that is close to reality and based on calculating the Signal to Noise Ratio (SNR).

In our network model, each node moves randomly within the network range speed rate, and to each is assigned a destination and a mobility speed. The simulation parameters are listed in Table 1.

Table 1. Simulation parameters

\begin{tabular}{ll}
\hline \multicolumn{1}{c}{ Parameters } & \multicolumn{1}{c}{ Value } \\
\hline Operation mode & $802.11 \mathrm{a}$ \\
Number of nodes & $20 ; 100$ \\
Simulation time & 60 minutes \\
Routing protocols & DSR, OLSR, AODV, \\
Addressing mode & DSDV, ZRP, GRP \\
Mobility rate & $2 \mathrm{~m} / \mathrm{s} ; 10 \mathrm{~m} / \mathrm{s}$ \\
Simulation area & $1200 \mathrm{~m} * 1200 \mathrm{~m}$ \\
Node movement model & Random waypoint \\
Data rate (Mbps) & 11 \\
Bandwidth (Mbps) & 11 \\
Data packet size & $128 * 8$ bits \\
Transmit power (w) & 0.10 \\
Simulator & OPNET 14.5 \\
\hline
\end{tabular}

\subsection{Performance Metrics}

A comparison of different works in literature shows that there are many metrics that can be considered for routing evaluation in MANETs[1][2], in this work the performance of the simulated results is analyzed according to different performance metrics that are described as follows: 
a. Routing traffic: the number of packets in the network.

b. Delay: the time between transmission and reception of a packet, its calculated based on the following formula:

$T_{\text {avg }}=\frac{\pi \sum_{i=1}^{N_{r}}\left(H_{r}^{i}-H_{t}^{i}\right)}{N_{r}}$

With the following parameters:

$H_{r}^{i}$ : Transmission time of the packet $\mathrm{i}$

$H_{t}^{i}$ : Reception time of the packet $\mathrm{i}$

$N_{r}$ : Total number of packets received.

c. Throughput: the ratio of the number of packets sent to the total number of packets. The greater value of throughput means the better performance of the protocol, this metric can be defined as follows:

$T=\frac{\mathrm{L}-\mathrm{C}}{L} R f(\gamma)$

Where the parameters represent:

L : Packet length.

$\mathrm{C}:$ Cyclic Redundancy Check.

$\mathrm{R}(\mathrm{b} / \mathrm{s})$ : Binary transmission rate.

$f(\gamma)$ : Packet success rate.

d. Mobility: shows the movement of nodes, it can be low or high, its calculation is made by measuring the movement of a node relatively to another.

\section{RESULTS AND ANALYSIS}

In this part, we present the obtained results through our model, based on evaluating metrics of traffic load, delay and throughput. In order to have relevant results, we choosed to evaluate at least one protocol from each routing protocol class of our classification (Figure 3.) Our simulation is divided into two scenarios of experiments:

\subsection{First Scenario: Low Node Mobility and Low Network Density}

In the first scenario, we deployed 20 mobile nodes with a mobility speed of $2 \mathrm{~m} / \mathrm{s}$ along with the other simulation parameters mentioned in Table 1.

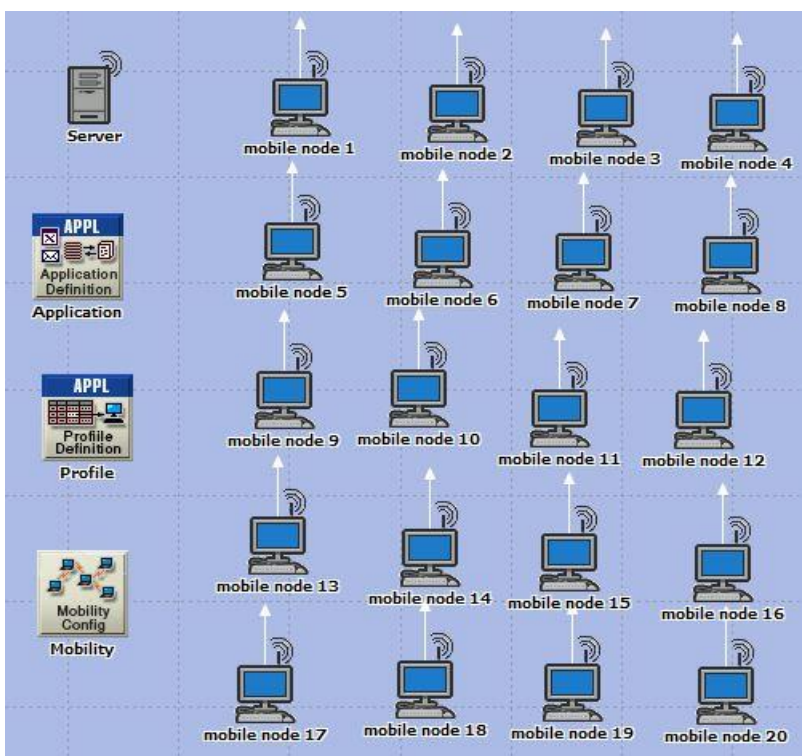

Figure 4. Simulation environment of the scenario 1 in OPNET 


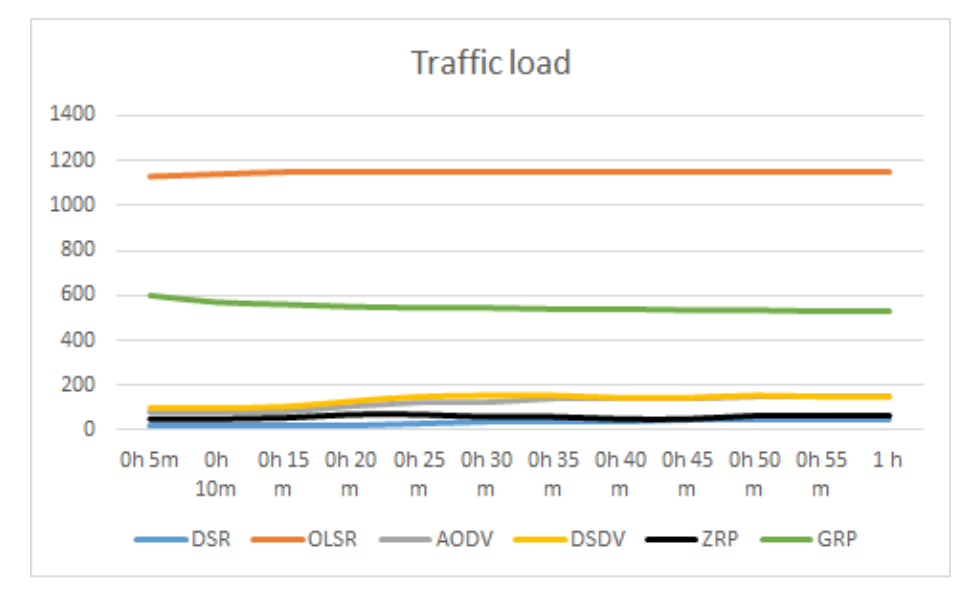

Figure 5. Evaluation of traffic load in scenario 1

In Figure 5. we compare the average of the traffic load between the mentioned routing protocols, it can be seen that DSR[13][14] shows the lowest traffic load followed by GRP[16] and AODV[6][11] while OLSR[11] shows the highest level of the traffic load.

DSR[13][14] generates less routing traffic, as the network is small, the prevalence of broken links is a factor that does not have much influence. OLSR[11] generates more communication overhead and takes more maintenance time due to the fact of being a link state protocol that uses a table-driven approach, while ZRP[13] uses the proactive approach for communication with zones and the reactive approach for the routes establishing

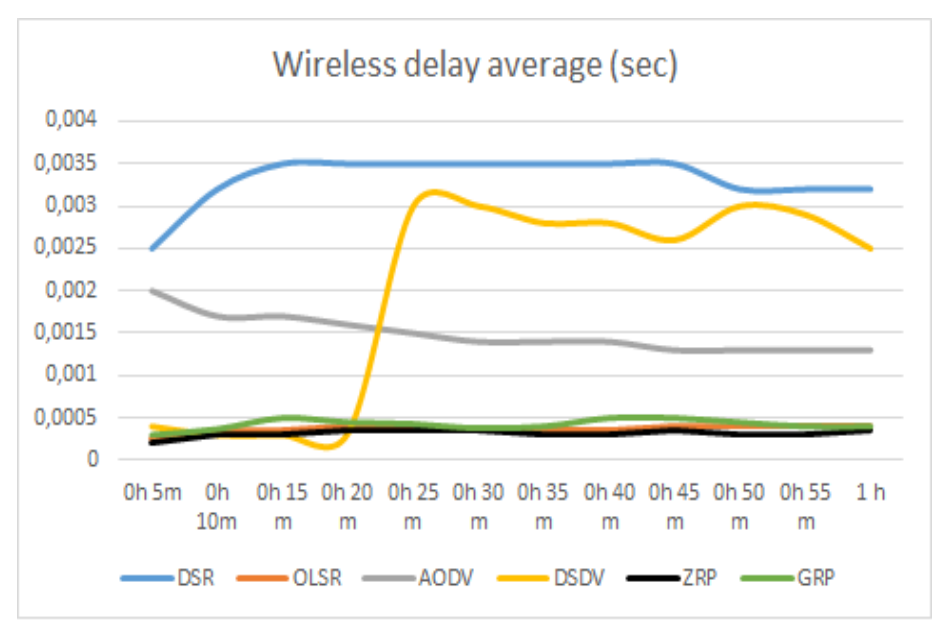

Figure 6. Evalution of wireless delay in scenario 1

In Figure 6. ZRP[13] protocol shows the lowest delay followed by OLSR[11] and GRP[16] while the DSR[13][14] shows the highest delay.

DSR[13][14] uses cached routes and most often, it sends the traffic on the obsolete routes which can cause retransmissions and excessive delays. Thus, in networks with high traffic sources, increasing the number of connections generates worse delay. On the other hand, the DSR[13][14] protocol attempts to minimize the effect of obsolete routes by using multipath links. In its turn, since DSDV[11] is based on periodic broadcasts it takes more time to converge before using a route. 


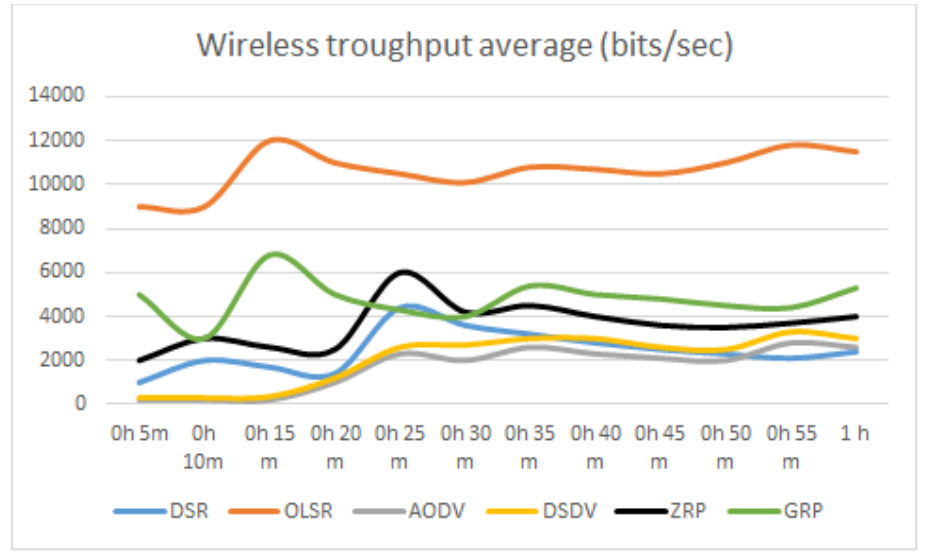

Figure 7. Evaluation of wireless throughput in scenario 1

From the Figure 7. we observe that OLSR[11] shows the highest throughput followed by GRP[16] and ZRP[13] while AODV[6][11] shows the lowest throughput. As OLSR[11] is a proactive routing protocol, the paths are readily available for traffic, this protocol maintains consistent paths in the network resulting in a low delay and in a higher throughput.

\subsection{Second Scenario: High Node Mobility and High Network Density}

In this scenario, we use the same simulation environment and we change the number of mobile nodes to 100 and the mobility speed to $10 \mathrm{~m} / \mathrm{s}$.

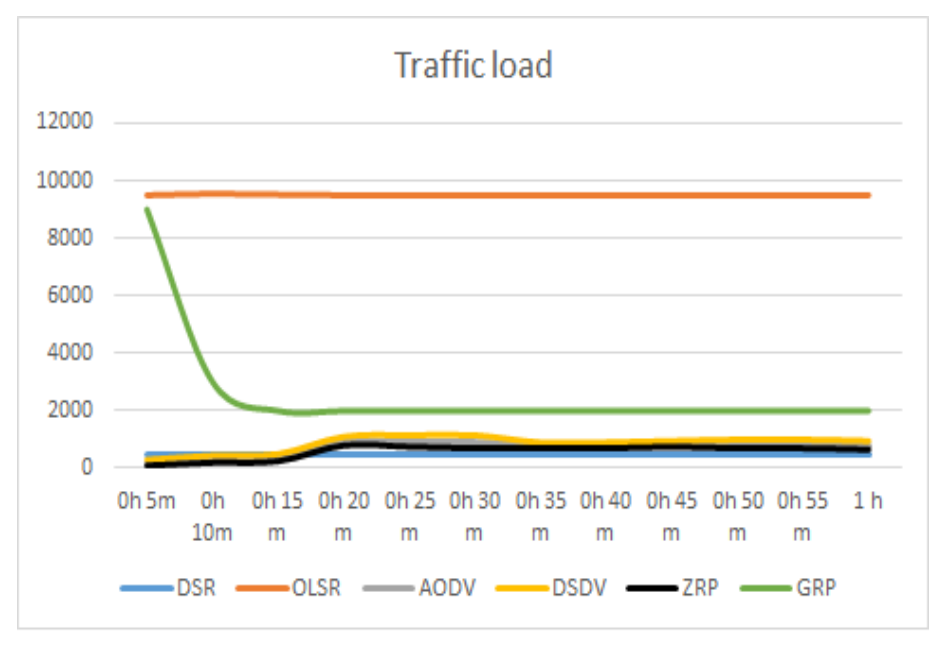

Figure 8. Evaluation of traffic load in scenario 2

It can be seen from Figure 8. that OLSR[11] generates the maximum traffic load followed by GRP[16] and DSDV[11] while DSR[13][14] generates the lowest traffic load. In the GRP[16] protocol, the traffic routing decreases hardly then remains almost stable during the rest of the simulation. 


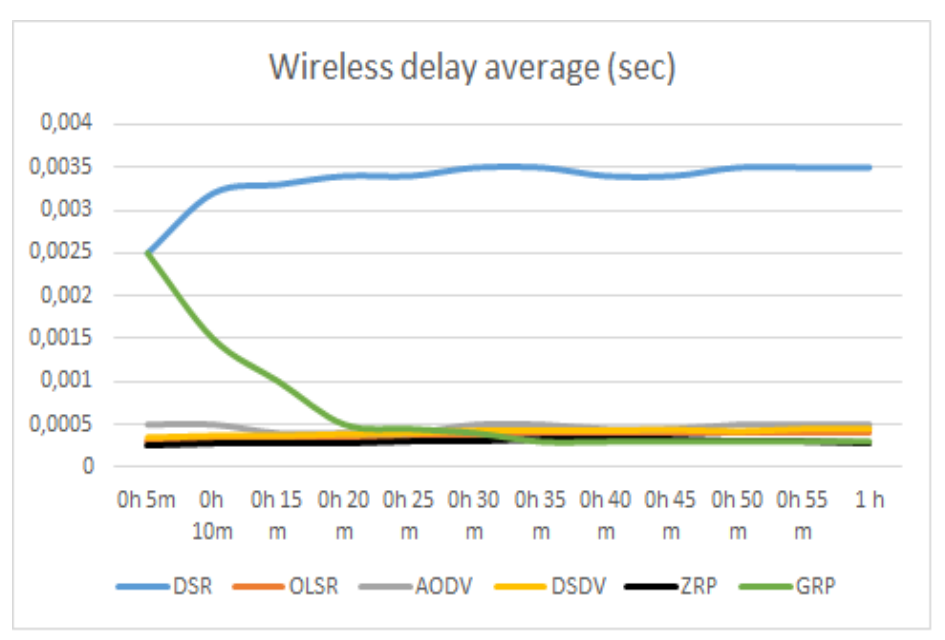

Figure 9. Evalution of wireless delay in scenario 2

According to the Figure 9. we observe that OLSR[11] has one of the lowest delay. OLSR[11] is a proactive routing protocol, which means that the network connections are always ready whenever the application layer has traffic to transfer. The periodic updates keep the routing paths available for use and the absence of a high latency induced by the discovery process routes in OLSR[11] explains its relatively low delay.

With a larger number of mobile nodes, the performances of OLSR[11] compete with those of AODV[6][11]. It can also be observed that GRP[16] offers an adequate delay with high number of nodes.

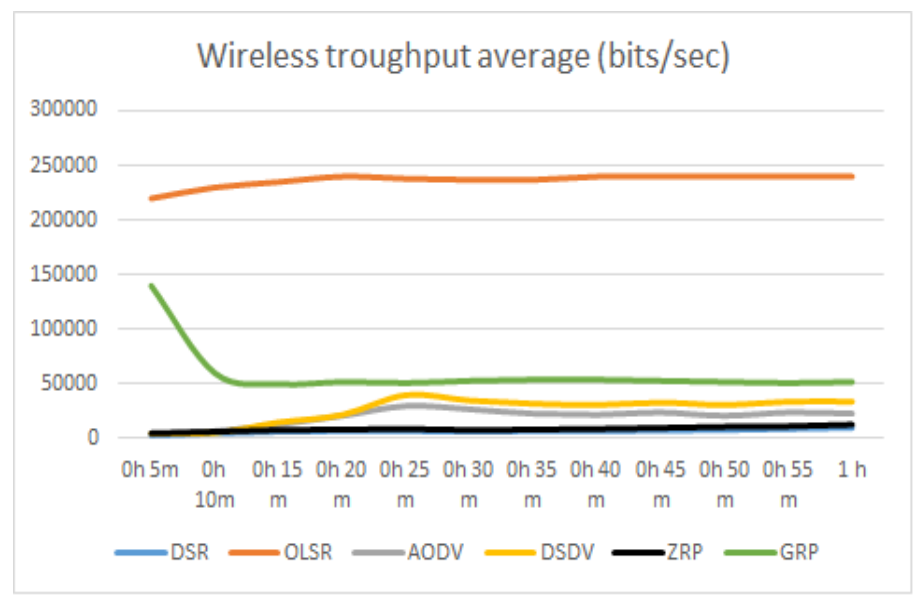

Figure 10. Evaluation of wireless throughput in scenario 2

In this scenario of simulation, we see that the throughput of AODV[6][11] drops fast because the available bandwidth is used mostly in the route searching mechanism, while protocols ZRP[13] and DSR[13][14] offer a very low throughput when comparing to the other protocols.

In conclusion, we observed that OLSR[11] shows a very low delay in both scenarios while AODV protocol[6][11] offers a better delay when the network size increases, concerning the OLSR protocol[11], mobility does not affect the density of traffic, but it increases with the network load and is almost at the same level in both scenarios. It is also concluded that DSR protocol[13][14] performs better in the case of small networks at any mobility speed and that the AODV protocol[6,11] gives its best results in networks with a relatively high number of traffic and high mobility speed. The OLSR protocol[11] has a constant throughput in both cases of mobility speed, as described above, OLSR is a proactive protocol that manages the consistent routing tables offering a coherent delay and this demonstrates its overall superiority. It should be noted that 
when the network size increases, OLSR routing tables[11] can become too large and this causes network congestion and leads to a degradation of the performance.

Table 2. Conclusion of routing protocols evaluation

\begin{tabular}{|c|c|}
\hline Routing Protocol & Conclusions \\
\hline$\overline{D S R}$ & $\begin{array}{l}\text { Very suitable for low density networks. Therefore, } \\
\text { its use in IPv6 environments would lead to higher } \\
\text { routing traffic. }\end{array}$ \\
\hline OLSR & $\begin{array}{l}\text { Very adequate to high broadband networks, the high } \\
\text { routing traffic generated shows that OLSR is not } \\
\text { suitable for low-density networks. }\end{array}$ \\
\hline AODV & $\begin{array}{l}\text { Suitable for low and medium density networks with } \\
\text { low mobility speeds. }\end{array}$ \\
\hline DSDV & $\begin{array}{l}\text { Slow protocol since it uses a periodic and event- } \\
\text { based update which causes excessive control in the } \\
\text { communication. }\end{array}$ \\
\hline ZRP & $\begin{array}{l}\text { The value of the area radius determines the } \\
\text { performance of the ZRP protocol, this value should } \\
\text { be as small as possible for better performance. }\end{array}$ \\
\hline GRP & $\begin{array}{l}\text { Reduces significantly the signalization (control } \\
\text { packets) especially in large and dynamic networks }\end{array}$ \\
\hline
\end{tabular}

\section{CONCLUSION}

In this article, we investigated the study of mobile ad hoc networks performance considering two key parameters that influence their implementation: the density of the network and the nodes mobility rate with a focus on the primordial problem of data routing. The different classes of routing protocols have been reviewed: proactive, reactive, hybrid, hierarchical and geographic to evaluate the most flexible ones and to determine the best context for their use.

The results show the relevance of our simulation model noting that the design of any routing protocol for mobile ad hoc networks must consider the physical limitations imposed by the mobile environment to avoid the degradation of the system performance. For the prospects of this work we intend to improve existing routing strategies, and to propose new routing protocols and solutions to the emergent needs in these networks.

\section{REFERENCES}

[1] Maude Si Liua; Peter Csaba Ölveczkyb; José Meseguera , "Modeling and analyzing mobile ad hoc networks in Real-Time," Journal of Logical and Algebraic Methods in Programming , vol 85,no.1, pp.34-66, 20160.

[2] Thodeti Srikanth; V.B. Narsimha , "Simulation-based approach to performance study of routing protocols in MANETs and ad-hoc Networks," International Journal of Computer Science and Network Security, vol.11, no.9, 2011.

[3] Y. Lin; Y. Chen; S. Lee , "Routing Protocols in Vehicular AdHoc Networks: A Survey and Future perspectives," Department of Computer Science and Information Engineering National Taipei University, Taipei, Taiwan, R.O.C.

[4] R. Verdone , "Wireless Sensor Networks," Proceedings of the 5th European Conference EWSN , Bolgna, 2008.

[5] F. J. Ros; P. M. Ruiz , "Efficient Gateway Discovery Algorithms for Delay-tolerant and Delay-constrained Data Traffic in Vehicular Ad-hoc Networks," In the IEEE Vehicular Technology Conference , pp.709-714, 2010.

[6] Sunita Sharma; Shruti Thapar , "Comparative Performance Analysis of AODV, DSDV and OLSR Routing Protocols in MANET Using OPNET," International Journal of Novel Research in Computer Science and Software Engineering, vol.2, no.2, pp.57-65, 2015.

[7] R. Balakrishna et U; Rajeswar Rao , "Comparisons of SAODV and TAODV, DSR Mobile ad hoc network Routing Protocols. International Journal of Advanced Networking and Applications. 2010; 2(1): 445-451.

[8] Popat K.A; Priyanka Sharma; Hardik Molia , "A Study of Routing Protocols for MANETs. In: Satapathy S., Bhatt Y., Joshi A., Mishra D. (eds) Proceedings of the International Congress on Information and Communication Technology. Advances in Intelligent Systems and Computing," 438. Springer, Singapore.

[9] Parulpreet Singh; Ekta Barkhodia; Gurleen Kaur Walia , "Evaluation of various Traffic loads in MANET with DSR routing protocol through use of OPNET Simulator," Department of Electronics Communication, LPU, Phagwara Punjab, India.

[10] Marica Amadeo; Claudia Campolo; Antonella Molinaro," Forwarding strategies in named data wireless ad hoc networks: Design and evaluation," Journal of Network and Computer Applications, pp.148-158, 2015.

[11] R. Bansal; H. Goyal; and P. Singh, "Analytical study the performance evaluation of mobile ad hoc networks using AODV protocol," International Journal of Computer Applications , vol.14, no.4, pp.34 - 37, 2011.

[12] S. Taneja; A. Kush , "A survey of routing protocols in mobile Adhoc networks. International Journal of Innovation, Management and Technology, vol.1, no.3, pp.279-285, 2010.

[13] Saad M. Adam; Rosilah Hassan , "Delay aware Reactive Routing Protocols for QoS in MANETs," Journal of Applied Research and Technology, vol.11, no.6, pp.844-850, 2013. 
[14] Vishal Sharma; Harsukhpreet Singh; Mandip Kaur , "Vijay Banga. Performance evaluation of reactive routing protocols in MANET networks using GSM based voice traffic applications," International Journal for Light and Electron Optics , vol.124, no.15, pp.2013-2016, 2013.

[15] Baraa T. Sharef; Raed A. Alsaqour; Mahamod Ismail , "Vehicular communication ad hoc routing protocols: A survey," Journal of Network and Computer Applications, vol.40, pp.363-396, 2014.

[16] Yaser Khamayseh; Ghadeer Obiedat; Munner Bani Yassin, "Mobility and Load Aware Routing protocol for ad hoc networks," Journal of King Saud University - Computer and Information Sciences , vol.23, no.2, pp.105-113, 2011.

[17] X. Wang; Y. Yang; A. J. An , "Multi-Metric Routing Decisions in VANET," In the IEEE International Conference on Dependable, Autonomic and Secure Computing, pp.551-556, 2009.

[18] S. Wan; J. Tang; R.S Wolff , "Reliable Routing for Roadside to vehicle Communications in Rural Areas," In the IEEE International Conference on Communications , pp.3017-3021, 2008. 